\title{
1 The promiscuous and highly mobile resistome of a superbug.
}

2

3 Ismael Hernández-González ${ }^{1}$, Valeria Mateo-Estrada ${ }^{1}$, and Santiago Castillo-

4 Ramírez ${ }^{1 *}$.

5

6 Programa de Genómica Evolutiva, Centro de Ciencias Genómicas, Universidad

7 Nacional Autónoma de México, Cuernavaca, México.

8

$9 \quad$ *Corresponding author: Santiago Castillo-Ramírez

10 E-mail address: iago@ccg.unam.mx

11

12

13

14

15

16

17

18

19

20

21

22

23

24

25

26

27

28

29

30

31 


\section{Abstract}

33 Antimicrobial resistance $(\mathrm{AR})$ is a major global threat to public health. Understanding

34 the population dynamics of $A R$ is critical to restrain and control this issue. However, no

35 study has provided a global picture of the resistome of Acinetobacter baumannii, a very

36 important nosocomial pathogen. Here we analyze 1450+ genomes (covering $>40$

37 countries and $>4$ decades) to infer the global population dynamics of the resistome of

38 this species. We show that gene flow and horizontal transfer have driven the

39 dissemination of $\mathrm{AR}$ genes in $A$. baumannii. We found considerable variation in $\mathrm{AR}$

40 gene content across lineages. Although the individual AR gene histories have been

41 affected by recombination, the AR gene content has been shaped by the phylogeny.

42 Furthermore, many AR genes have been transferred to other well-known pathogens,

43 such as Pseudomonas aeruginosa or Klebsiella pneumoniae. Finally, despite using this

44 massive data set, we were not able to sample the whole diversity of AR genes, which

45 suggests that this species has an open resistome. Ours results highlight the high

46 mobilization risk of AR genes between important pathogens. On a broader perspective,

47 this study gives a framework for an emerging perspective (resistome-centric) on the

48 genome epidemiology (and surveillance) of bacterial pathogens.

50 Keywords: antibiotic resistance; resistome; horizontal gene transfer; population

51 genomics; genome epidemiology; A. baumannii. 


\section{Introduction}

64 Antimicrobial resistance is a major global menace to humans all over the world. In this regard, the ESKAPE (Enterococcus faecium, Staphylococcus aureus, Klebsiella pneumoniae, Acinetobacter baumannii, Pseudomonas aeruginosa, and Enterobacter species) group is a significant cause of deaths and burden disease in many countries ${ }^{1}$. This group of bacteria can easily acquire antimicrobial resistance genes (ARGs). In 2017 the World Health Organization issued a list of bacterial pathogens for which novel drugs are immediately required ${ }^{2}$. At the top of this list, having the highest priority status (priority 1: critical), was carbapenem-resistant $A$. baumannii. Notably, most $A$. baumannii hospital infections are caused by multidrug resistant (MDR) isolates.

The resistome is the set of ARGs present in a given species or a particular environment ${ }^{3}$. Over the last decade the resistome of some species and some ecological communities have been studied to an unprecedented detail due to the extensive amount of genetic information provided by genome sequencing. For instance, metagenomics and functional genomics studies have shown that ARGs are frequently found in many different environments ${ }^{4-8}$. These go from human-made environments, such as hospitals ${ }^{5}$, to pristine and even isolated environments, such as isolated caves 7. Furthermore, many ARGs are globally distributed and are rather diverse. Additionally,

82 population genomics and genomic surveillance studies have been very useful to study

83 the geographical and temporal spreading of important MDR lineages ${ }^{9-14}$. However,

84 most of these studies have used the core genome as the framework to understand the dynamics of the ARGs. Indeed, ARGs are often just mapped onto the core phylogeny.

86 This strategy can be useful for rather clonal species, such as Staphylococcus aureus.

87 Nonetheless, in species with high rates of gene content variation, such as $A$. baumannii ${ }^{15}$, this strategy is bound to be problematic. Furthermore, ARGs are commonly found within mobile genetic elements. Hence, ARGs can be easily moved horizontally within and between species. In this regard, the potential transfer of ARGs between pathogens

91 from different genera is especially concerning in hospital settings ${ }^{6}$. Therefore, to

92 properly track the transmission dynamics of ARGs, population genomics studies

93 focusing on the ARGs per se are required. 
95 Over the last decade, and due to its clinical relevance, a lot of knowledge has been gained for $A$. baumannii through the genome sequencing of many hundred isolates.

97 However, despite the rapid accumulation of genomes, there has not been a single study

98 that has tried to use all this information to comprehensively study the population 99 dynamics of the resistome of this species. Here we produce the most extensive view of

100 the resistome of $A$. baumannii. The data set here amassed has almost 1500 high-

101 quality genomes, covers 42 countries and four decades, and contains 149 lineages

102 (Sequence Types). Our results showed that this is a very dynamic resistome, showing

103 high levels of gene flow within the global population of $A$. baumannii. Furthermore,

104 many ARGs are exchanged with other distantly related bacterial pathogens.

\section{Results}

107 The vast accessory resistome of $\boldsymbol{A}$. baumannif. To have the most comprehensive 108 picture of the resistome of $A$. baumannii, we included as many genomes as possible; 109 however, we did pay attention not to include lower quality data. Thus, only genomes 110 showing a high-quality assembly were downloaded (see methods). Furthermore, we 111 included only complete and uncontaminated genomes (completeness $\geq 95 \%$ and

112 contamination $\leq 5 \%$, see methods). We also corroborated that all the genomes

113 belonged to $A$. baumannii via an Average Nucleotide Identity (ANI) analysis; where only

114 isolates having $\geq 95 \%$ identity with the type strain ATCC19606 were considered. We 115 kept a total of 1472 genomes for downstream analyses (listed in Supplementary Table 116 1). This is the most extensive data set ever created for this species: it covered isolates

117 from 42 different countries (Figure 1a), a period of time spanning 76 years (four 118 decades without outliers), and 149 Sequence Types (Figure 1b). Then, we used the 119 Comprehensive Antibiotic Resistance Database ${ }^{16}$, which is rigorously curated and 120 monthly updated, to catalogue and quantify the ARGs in the 1472 genomes. We found 121 that the average number of ARGs per genome was 29.38; the frequency distribution 122 histogram of ARGs per genome is presented in Figure 2a. The ARGs were classified in 123199 ARG families and these covered a wide range of drug classes (see Table 1 and 124 Supplementary Table 2). We noted that no single group was present in all the genomes 
125 (see Table 1). Twelve ARG families were present in more than $95 \%$ of the genomes.

126 These gene families correspond to the RND efflux pumps, well-known intrinsic

127 resistance genes, and were present in most countries and in almost all the STs (see

128 Table 1). We also found that 26 ARG families (13\%) were present in less than 95\% of

129 the genomes but in more than $15 \%$ of the genomes (see Table 1). Remarkably, almost

130 all of these 38 most frequent ARG families were affected by either recombination or

131 horizontal gene transfer (see Table 1). However, the vast majority of the ARG families

132 (161 groups, 81\%) were contained in less than $15 \%$ of the genomes (see

133 Supplementary Table 2). These ARG families were present in few countries and few

134 STs (see Supplementary Table 2). Taken together, these results show that a

135 considerable amount of ARGs are found within these genomes. Nonetheless, these

136 ARGs do not belong to the core genome and are not strictly vertically transmitted.

137 Notably most of them are just present in less than $15 \%$ of the strains.

High gene flow within the species and HGT with other pathogens. The fact that most of the ARGs are just present in some of the genomes implies that ARGs are

141 frequently lost and gained. In accordance with this, we recently showed that gene

142 turnover was of paramount importance in a recently emerged lineage of this species ${ }^{15}$.

143 To further explore this, we analyze ARG content variation within individual STs; we only

144 considered those STs that had 10 or more genomes per ST. This approach allowed us

145 to analyzed ARGs variation over short timescales. If ARGs were to be only

146 disseminated by clonal expansions (without HGT or gene loss), the amount of ARGs

147 per genome would be the same for a given ST. Contrary to this, Figure $2 b$ clearly shows

148 that there is a huge variation, not only within STs but also between STs (Kruskal-Wallis

149 test, $\mathrm{p}$-value < 2.2e-16). While the ST that had most ARGs was ST229 with an average

150 number of 32 ARGs per genome, the ST with the lowest mean number was ST447 (see

151 Figure 2b) with 20 ARGs per genome. Thus, this high variation in ARG content within

152 STs implies that, even at short timescales, acquisitions and losses of ARGs are very

153 common. Then we went on to look if any of these ARG families have been horizontally

154 transferred very recently (see methods). We noted that 78 ARG families (39\%) had

155 identical allelic variants in other bacteria (see Table 1 and Supplementary Table 2). 
156 Notably, most the HGT events were located in other nosocomial pathogens (see

157 Supplementary Table 2 and Figure 3a); Klebsiella pneumoniae and Pseudomonas

158 aeruginosa are two of the most striking cases. Collectively, these results show that HGT

159 (and gene loss) are of paramount importance not only within the species but also with

160 other (nosocomial) bacterial species.

\section{An open resistome with ARG content structured by the phylogeny.}

163 Although ARGs are subject to loss and gain processes, the presence of the ARGs could

164 be structured by the phylogeny. This is because, when considering the accessory

165 genome, HGT between closely related lineages is likely to be more successful; also, the

166 more distantly related two lineages are, the higher the likelihood of gene loss. Thus, to

167 explore this, we conducted pairwise comparisons of all the isolates correlating how

168 similar they were in terms of their ARGs versus their ANI values. We used ANI as a

169 proxy for the phylogenetic relationship of the isolates. To establish how similar the

170 ARGs profiles between the isolates were, we used the Jaccard index. A perfect

171 correlation would imply that the gains and losses of ARGs are shaped by phylogeny.

172 Although not very strong, the Spearman's correlation was significant $\left(r^{2}=0.57, p\right.$-value <

$1732.2 \mathrm{e}-16)$. Thus, there is a correlation between ANI values and similarity in the ARGs

174 profiles, which implied that ARGs content variation correlated with the phylogeny.

175 Finally, we wanted to establish if we were able to sample the diversity of ARGs in this

176 species. Of note, given the very extensive nature of this data set in temporal and

177 geographical terms, we would expect so. We ran an accumulation curve analysis to

178 evaluate this (see methods and Figure 3b). This analysis showed that we have

179 sampled much of the ARG family diversity. However, the curve did not level off and the

180 slope is still very steep in the last section of the curve. Thus, the discovery rate of ARGs

181 is still high after almost 1500 genomes and a lot more ARGs remain to be found. Taken

182 together, these results imply that although gene flow is common within ARGs, ARGs

183 content variation within the isolates is structured to some extent by the phylogeny.

184 Furthermore, this seems to be an open resistome. 


\section{Discussion}

188 Metagenomics studies over the last decade have been of paramount importance to 189 characterize the resistome dynamics in different environments. However, reliable

190 population genomic studies are still very much required to properly describe the 191 transmission dynamics of ARGs both within and between bacterial species. A clear 192 understanding of these transmission dynamics is essential for the effective use of 193 antibiotics. Notably, this will provide useful knowledge to inform public health actions 194 and infection control teams to establish better treatment options. However, there has not been a single study on the resistome of $A$. baumannii at global scale. Here we 196 gathered the most extensive - in terms of geographic and temporal components 197 dataset ever created for this important nosocomial pathogen. Our analyses underscore 198 a highly mobile and even promiscuous resistome of $A$. baumannii. We found a 199 considerable variation in ARGs profiles among the different lineages (STs). On top of 200 that, we noted that many ARGs have been exchanged with other well-known 201 pathogens.

In some important bacterial pathogens, such as K. pneumoniae, MDR isolates (showing many ARGs) are frequently found in high-risk clones. On the contrary, in A. baumannii the ARGs are well disperse among the global population and clonal expansion does not seem to be the major force dispersing the ARGs. Previous studies have shown that gene content variation is of paramount importance for this pathogen ${ }^{15}$. Importantly, even very recently emerged ARGs seems to have experienced HGT between different lineages of this species ${ }^{13}$. One of the most relevant findings of our study, from a clinical

210 microbiology point of view, is that $A$. baumannii readily exchanges ARGs with other

211 highly critical pathogens such as K. pneumonia or Pseudomans aeruginosa. In this

212 respect, a recent study also found many instances of HGT across MDR bacteria from 213 different genera in a single hospital ${ }^{17}$. In connection with the previous points, infection

214 control and detection strategies considering this pathogen should be focused on the 215 ARGs, rather than on particular lineages, to prevent the transmission of such genes.

216 Thus, the risk of ARGs across important nosocomial pathogens should be considered of 217 paramount importance not only from a microbial genomics point of view but also from 
218 infection control perspective. Finally, and connected with the previous point, we

219 observed that, notwithstanding the vast data set we used, our accumulation curve

220 analysis implies that we did not sample the full diversity of ARGs within A. baumannii.

221 Thus, very likely this species has an open resistome; given the tendency of this species

222 acquires ARGs from other bacteria an open resistome is not actually unexpected.

224 One of the limitations of our study is that we underestimated the rate of HGT with other

225 bacteria. This is because we only considered very recent HGT events, i.e., identical

226 gene sequences in different bacteria. Clearly, the rate of HGT is bound to be

227 considerably higher, if HGT events of different ages are to be included in the estimation.

228 Another limitation of our study is that our analyses were based on a genetic definition of

229 antibiotic resistance ${ }^{18}$. We acknowledged that further studies considering also

230 microbiological and clinical definitions of antibiotic resistance are required to fully grasp

231 the complex issue of antimicrobial drug resistance. Nonetheless, understanding the

232 populations dynamics of the ARGs is crucial for the tracking and surveillance of ARGs

233 not only in the clinic but also in non-clinical settings. In this regard, we recently show

234 that environmental isolates of $A$. baumannii can be an important source of ARGs ${ }^{19}$.

235 Without doubt, the transmission dynamics of ARGs can be used to implement ad hoc

236 infection-control actions within different health systems all over the world. Thus, similar

237 studies are required for many other members of the ESKAPE group, or any other

238 relevant human pathogen for that matter, to properly tackle the major problem of

239 antimicrobial drug resistance.

\section{Methods}

\section{Genomes and quality check}

243 Genomes sequences were downloaded in early March 2020 from the Refseq NCBI

244 Reference Sequence Database. Only genomes with reliable quality genome assembly

245 status were considered; thus, only genomes showing an assembly level of "complete

246 genome", chromosome and scaffold were included in the final data set. We also used

247 CheckM $^{20}$ to evaluate the completeness and contamination of the genomes and only

248 genomes showing $95 \%$ (or higher) of completeness and equal or less than $5 \%$ of 
contamination were considered for downstream analyses. For consistency all the genomes were re-annotated with $\mathrm{PROKKA}^{21}$ and the final list of the genomes included is provided in Supplementary Table 1.

252

\section{Average nucleotide identity analysis and ST assignation}

254 To make sure that all genomes belong to Acinetobacter baumannii and to evaluate how 255 similar were among them, we conducted an Average Nucleotide Identity (ANI) analysis 256 using OrthoANI ${ }^{22}$. Using pairwise comparison, every genome was compared to rest of

257 the genomes and to the type strain ATCC 19606 (Biosample accession number

258 SAMN13045090). We kept only the isolates that had an ANI value of $95 \%$ or higher

259 versus the type strain. We downloaded the allelic variants for profiles of all the ST

260 described thus far for both the Oxford and the Pasteur schemes from the PubMLST

261 database ${ }^{23}$. Then, we used blastn (requiring $100 \%$ identity) to assign an allelic profile to

262 each genome considering both schemes. Given that the Oxford MLST scheme has

263 some issues with paralogy in the locus gdhB, we processed the data as in ${ }^{23}$ to discard

264 the paralogous genes causing issues.

Antibiotic resistance gene prediction and pangenome analysis.

267 We employed the CARD database ${ }^{16}$ the infer the ARGs in all the A. baumannii

268 genomes. We used the Resistance Gene Identifier tool from CARD and only "Perfect"

269 and "Strict" cases were considered but we also required a $\geq 90 \%$ coverage between

270 the query and the target. Antibiotic drug class were also determined through CARD.

271 Then, the ARGs were assigned to the different types of genome categories as follows:

272 core genome, genes in 100\% of the isolates; soft core genome, genes between 95\%

273 and less than $100 \%$ of the isolates; shell genome, genes in less than $95 \%$ but greater

274 than $15 \%$ of the isolates; and cloud genomes, genes in less than $15 \%$ of the isolates.

275 The ARGs were grouped into ARG families using the CARD classification.

277 Recombination analysis, horizontal gene transfer with other species, and 278 accumulation curve analysis. 
279 Recombination analysis were run on all the ARG family groups that had at least 3 280 sequences and which shared at least $80 \%$ of coverage among all the sequences. We

281 used the PHIpack program ${ }^{25}$ that implements the "Pairwise Homoplasy Index" test (PHI

282 test) to detect recombination signals within the ARG families. To infer recent events of

283 HGT between the ARGs in A. baumannii and other bacteria, we used blastn (with an e-

284 value of $1 \mathrm{e}-50$ ) to search these ARGs against the "nt" database from the NCBI. Off 285 note, as we wanted only very recent HGT events, we considered only those hits that 286 were $100 \%$ identical (both in the coverage and in the \% of identity) to the query. As 287 query could have identical hits from several species, we chose that species that had 288 highest number of identical hits. We used the Vegan $\mathrm{R}$ library ${ }^{26}$ to run a rarefaction 289 curve of the number ARGs as a function of the number of genomes. We employed the 290 "specaccum" function for this, setting the method "rarefraction". The visualization tool 291 Circos ${ }^{27}$ was employed to illustrate the HGT cases between the ARGs in A. baumannii 292 and the other three major bacteria contributors.

\section{Acknowledgments}

295 We are very grateful to Alfredo José Hernández Álvarez and Victor Manuel del Moral 296 Chávez for installing several of the programs employed in this study. SCR is grateful to 297 his friend Timothy Read for reviewing the manuscript.

\section{Authors' contribution}

300 SCR conceived and supervised the study. VME conducted preliminary analyses of the antibiotic resistance gene prediction. ILHG carried out most of the analyses. SCR wrote 302 the manuscript and all the authors approved it.

\section{Conflicts of interest}

305 All the authors report no potential conflict of interest.

\section{Funding}

308 This study was supported by supported by "Programa de Apoyo a Proyectos de 309 Investigación e Innovación Tecnológica" (grant no. IN206019) and "Consejo Nacional 
310 de Ciencia y Tecnología” (CONACyT) Ciencia Básica 2016 (grant number 284276) to

311 SCR. VME is a PhD student from the Programa de Doctorado en Ciencias Biomédicas,

312 Universidad Nacional Autónoma de México, and is supported by a CONACyT

313 postgraduate fellowship. ILHG acknowledges support from UNAM Dirección General de

314 Asuntos de Personal Académico postdoctoral fellowship funding.

\section{References}

3171 De Oliveira, D. M. et al. Antimicrobial Resistance in ESKAPE Pathogens. Clinical

318 Microbiology Reviews 33 (2020).

3192 Organization, W. H. WHO publishes list of bacteria for which new antibiotics are

$320 \quad$ urgently needed. (2017).

3213 Waglechner, N. \& Wright, G. D. Antibiotic resistance: it's bad, but why isn't it worse?

$322 \quad$ BMC biology 15, 1-8 (2017).

3234 D'Costa, V. M., McGrann, K. M., Hughes, D. W. \& Wright, G. D. Sampling the antibiotic resistome. Science 311, 374-377 (2006).

5 Evans, D. R. et al. Systematic detection of horizontal gene transfer across genera among multidrug-resistant bacteria in a single hospital. Elife 9, e53886 (2020).

3276 Martínez, J. L., Coque, T. M. \& Baquero, F. What is a resistance gene? Ranking risk in resistomes. Nature Reviews Microbiology 13, 116-123 (2015).

3297 Pawlowski, A. et al. (2016).

3308 Perry, J. A., Westman, E. L. \& Wright, G. D. The antibiotic resistome: what's new? Current opinion in microbiology 21, 45-50 (2014). Castillo-Ramírez, S., Mateo-Estrada, V., Gonzalez-Rocha, G. \& Opazo-Capurro, A. Phylogeographical analyses and antibiotic resistance genes of Acinetobacter johnsonii highlight its clinical relevance. Msphere 5 (2020).

10 Challagundla, L. et al. Phylogenomic classification and the evolution of clonal complex 5 methicillin-resistant Staphylococcus aureus in the Western Hemisphere. Frontiers in Microbiology 9, 1901 (2018).

11 Frisch, M. et al. Invasive methicillin-resistant Staphylococcus aureus USA500 strains from the US Emerging Infections Program constitute three geographically distinct lineages. MSphere 3 (2018).

12 Goldstone, R. J. \& Smith, D. G. A population genomics approach to exploiting the accessory'resistome' of Escherichia coli. Microbial Genomics 3 (2017). resistant Acinetobacter baumannii isolates from tertiary hospitals in Honduras. Microbial Drug Resistance 25, 690-695 (2019). 
15 Graña-Miraglia, L. et al. Rapid gene turnover as a significant source of genetic variation

Alcock, B. P. et al. CARD 2020:

16 Alcock, B. P. et al. CARD 2020: antibiotic resistome surveillance with the comprehensive

17 Evans, D. R. et al. Systematic detection of horizontal gene transfer across genera among multidrug-resistant bacteria in a single hospital. Elife 9, doi:10.7554/eLife.53886 (2020).

18 Courvalin, P. Predictable and unpredictable evolution of antibiotic resistance. Journal of internal medicine 264, 4-16 (2008).

19 Hernández-González, I. L. \& Castillo-Ramírez, S. Antibiotic-resistant Acinetobacter baumannii is a One Health problem. The Lancet Microbe 1, e279 (2020). Parks, D. H., Imelfort, M., Skennerton, C. T., Hugenholtz, P. \& Tyson, G. W. CheckM: assessing the quality of microbial genomes recovered from isolates, single cells, and

21 Seemann, T. Prokka: rapid prokaryotic genome annotation. Bioinformatics 30, 20682069, doi:10.1093/bioinformatics/btu153 (2014).

22 Lee, I., Ouk Kim, Y., Park, S. C. \& Chun, J. OrthoANI: An improved algorithm and software for calculating average nucleotide identity. Int J Syst Evol Microbiol 66, 1100-1103, doi:10.1099/ijsem.0.000760 (2016).

23 Jolley, K. A., Bray, J. E. \& Maiden, M. C. Open-access bacterial population genomics: BIGSdb software, the PubMLST. org website and their applications. Wellcome open research 3 (2018).

24 Page, A. J. et al. Roary: rapid large-scale prokaryote pan genome analysis. Bioinformatics 31, 3691-3693, doi:10.1093/bioinformatics/btv421 (2015). the presence of recombination. Genetics 172, 2665-2681, doi:10.1534/genetics.105.048975 (2006). (2018).

37827 Krzywinski, M. et al. Circos: an information aesthetic for comparative genomics. Genome research 19, 1639-1645 (2009). 
FIGURES AND TABLE

Figure 1 - An extensive data set, covering many countries, years, and lineages.

(a) Countries from which the genomes were sampled; the color key gives the number of genomes per region. (b) Number of genomes per ST under the Oxford scheme; only STs with 2 or more genomes are shown.

Figure 2 - High variation of ARGs.

(a) Histogram of the number of ARGs per genome. (b) Boxplots showing the variation in the number of ARGs within and between STs; only STs with at least 10 genomes are 400 shown.

Figure 3 - Horizontal transfer of ARGs and an open resistome.

(a) Circos plot showing the cases of HGT between ARGs A. baumannii (the little (7 to 12 clockwise) involved in $97 \%$ of the HGT cases are shown on the left-hand side. (b) Accumulation curve of the number of ARGs as a function of the number of genomes.

407 The red area shows the confidence intervals.

Table 1 - The most frequent ARGs found in the species Information for the most frequent ARGs is provided. These ARGs were present in more than 100 genomes. The number of genomes, countries and ST in which every ARG was present is reported. In bold are those ARGs that had recombination signals and the superscript ${ }^{\mathrm{H}}$ means that identical allelic variants were found in other bacteria. 


\section{SUPPLEMENTARY MATERIAL}

422 Supplementary Table 1

423 List of the genomes along with their metadata employed in this study.

424

\section{Supplementary Table 2}

426 Information of all the 199 ARGs found in this study. The number of genomes, countries

427 and STs are provided; as well as whether the ARG was subject to HGT.

428

429 
A

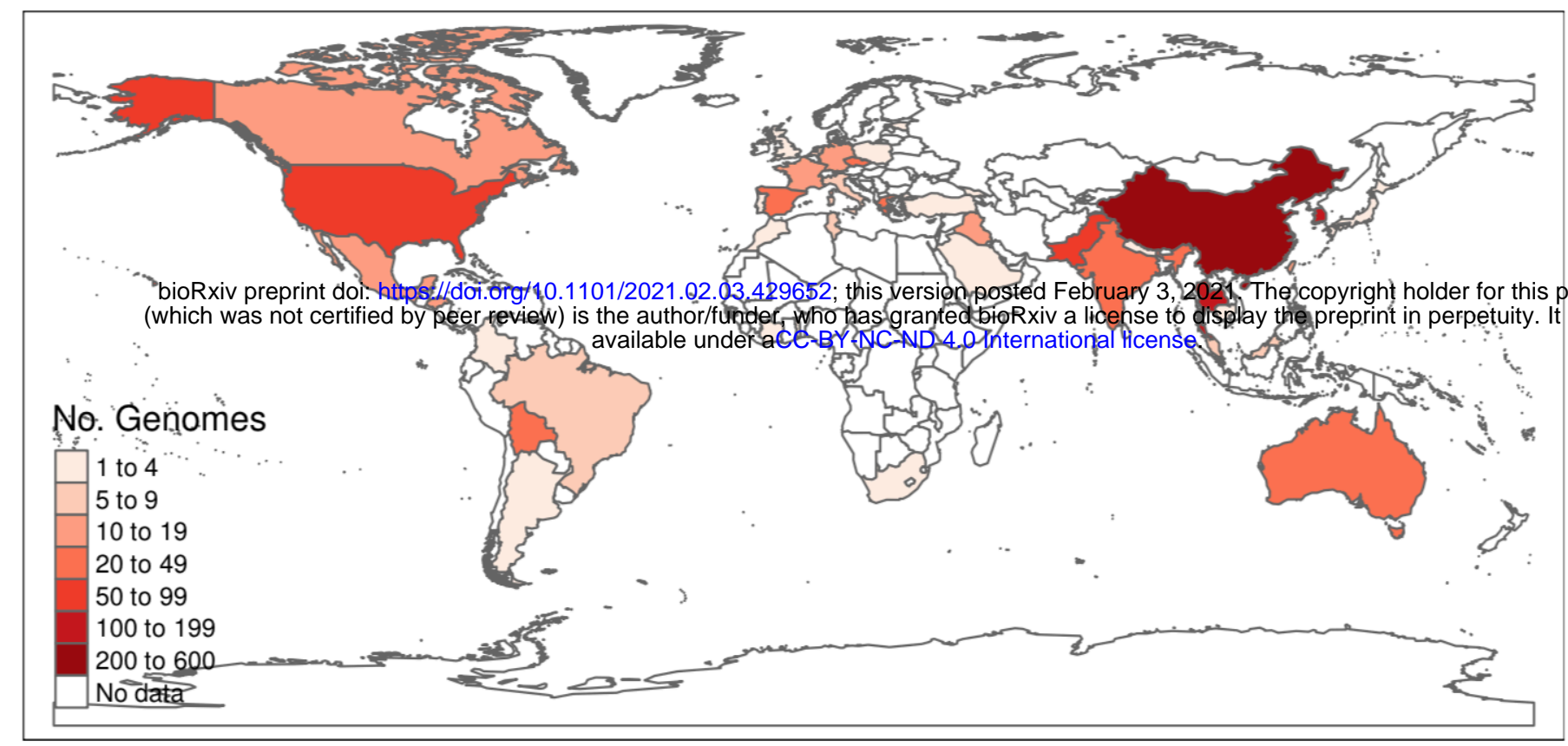

B

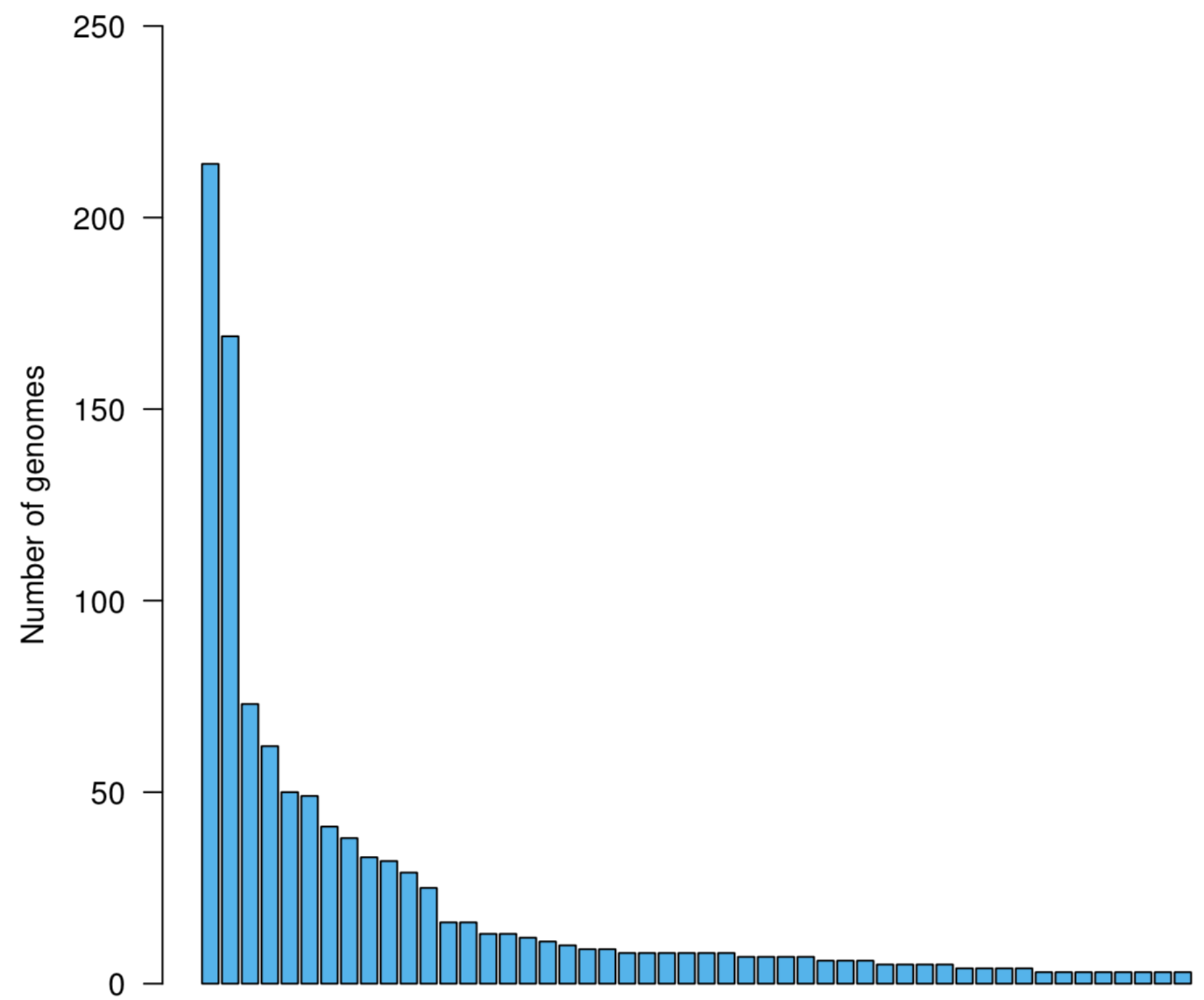

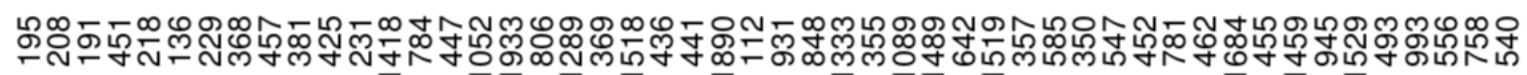


A

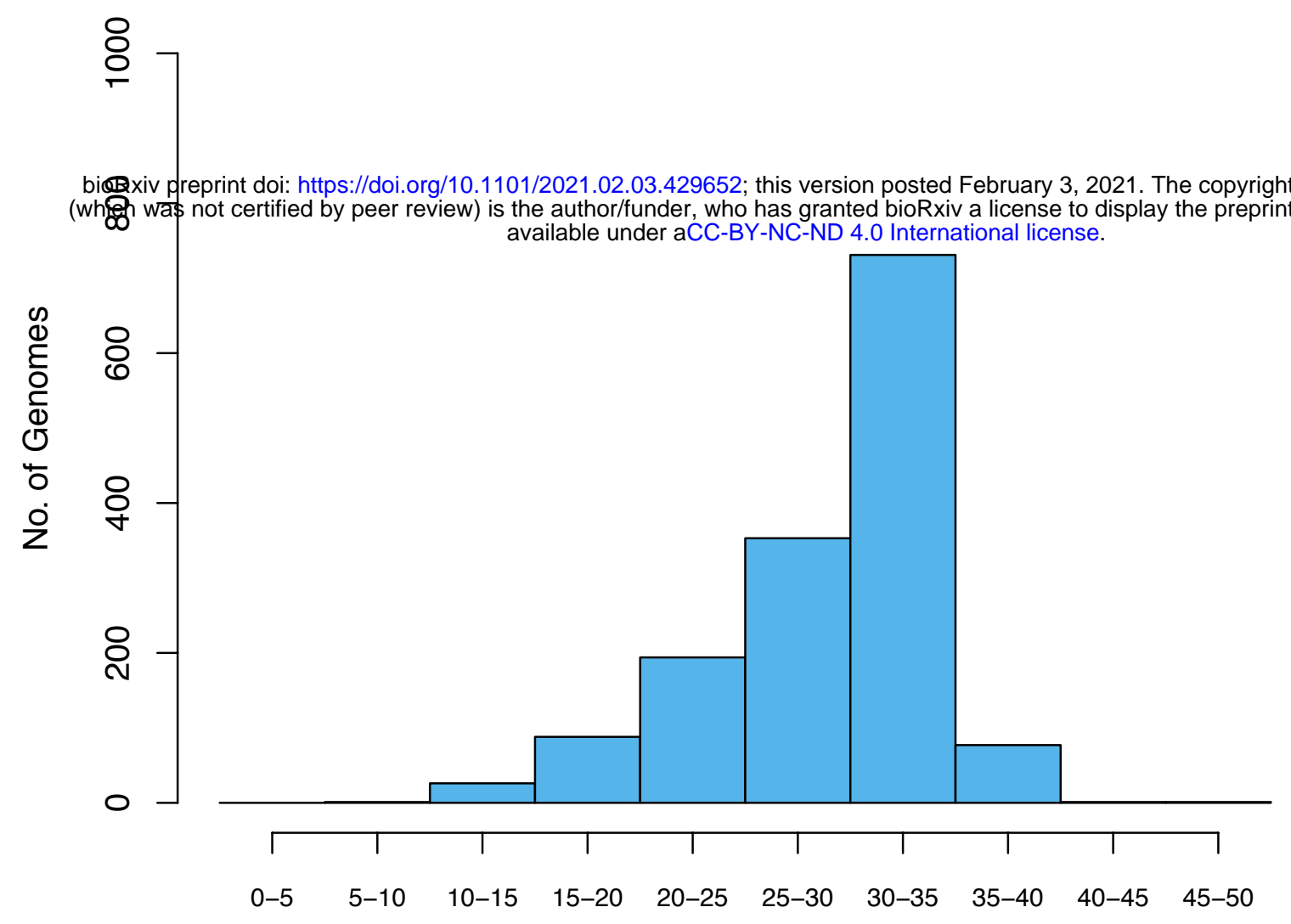

B

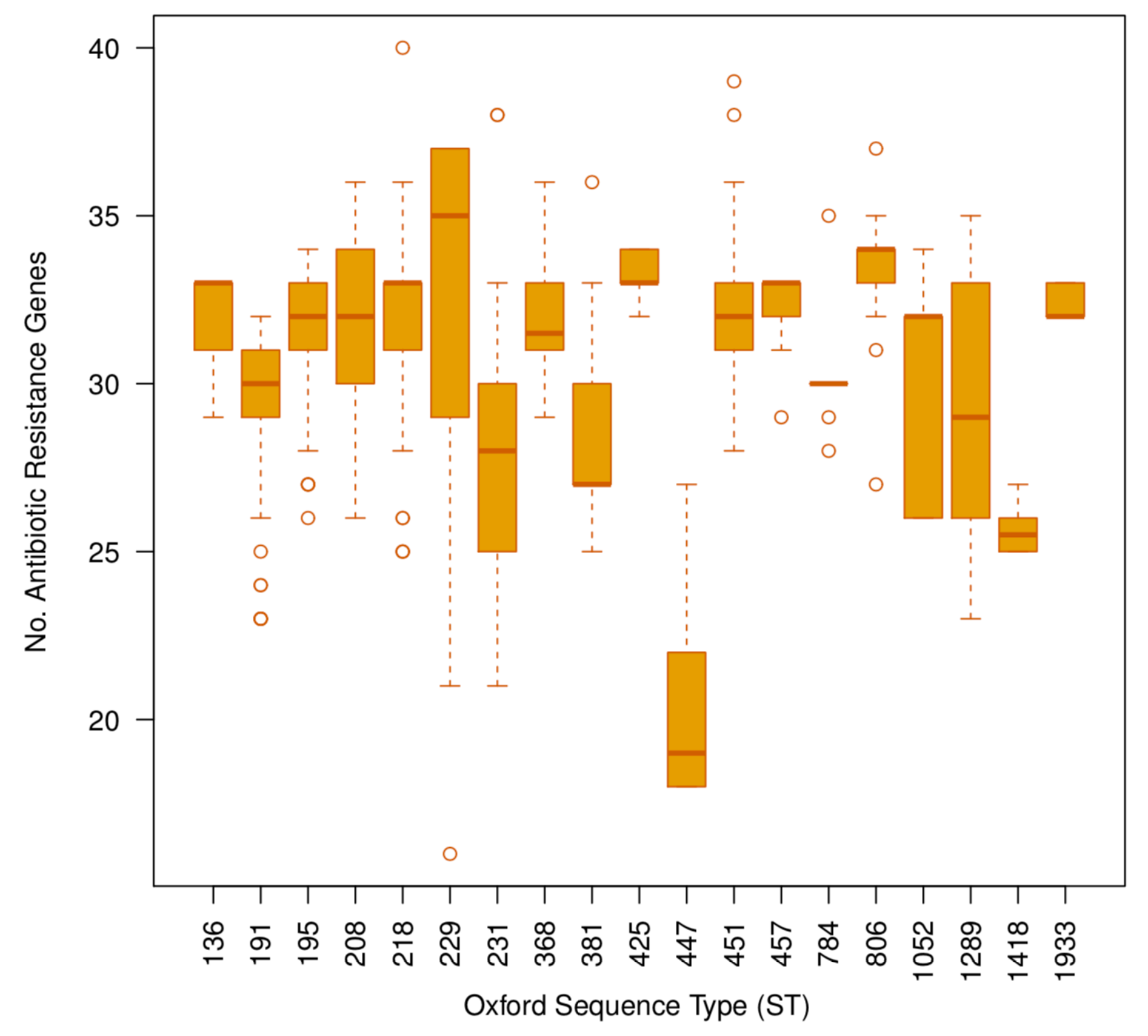


A

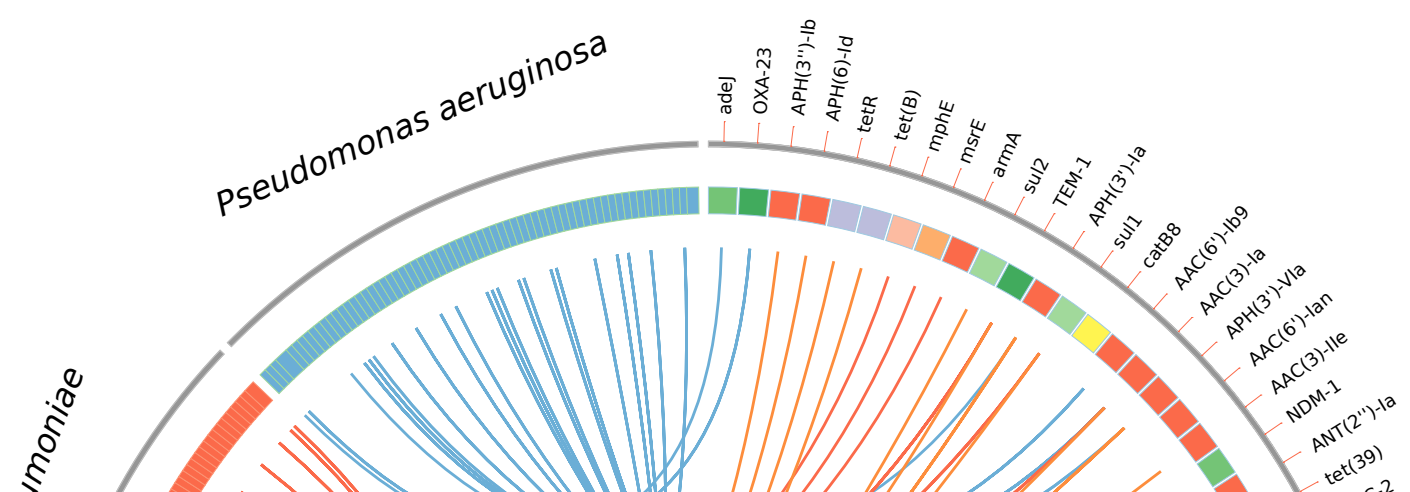

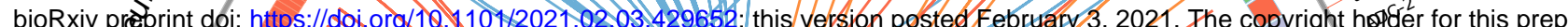
(which was not certified by peer review) is the author/funder. who has granted bioRxiv a lieense to display the preprint in pezpetuity. It is $\mathrm{m}$
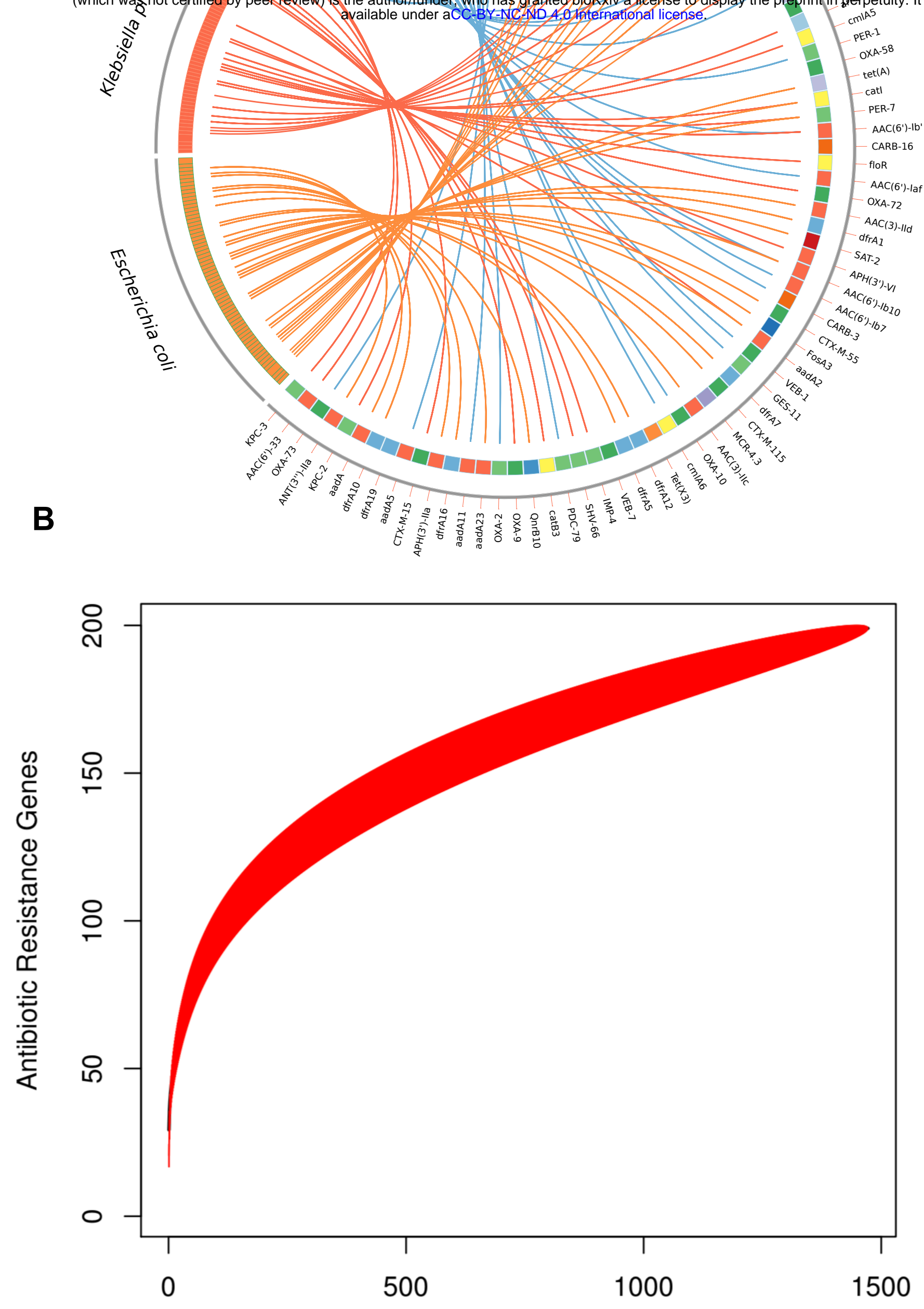

Number of Genomes 